\title{
A Study on C-E Translation of Scenic Spots Names under Creative Treason
}

\author{
Wenmin Li, Yu Wang \\ Shaanxi University of Science and Technology, College of Arts and Sciences, Xi'an, China \\ Email: 18437963106@qq.com
}

How to cite this paper: Li, W.M. and Wang, Y. (2021) A Study on C-E Translation of Scenic Spots Names under Creative Treason. Open Access Library Journal, 8: e8174. https://doi.org/10.4236/oalib.1108174

Received: November 22, 2021

Accepted: December 5, 2021

Published: December 8, 2021

Copyright $\odot 2021$ by author(s) and Open Access Library Inc.

This work is licensed under the Creative Commons Attribution International License (CC BY 4.0).

http://creativecommons.org/licenses/by/4.0/

\begin{abstract}
State Chinese and English Bilingual landscape is required in national five-star scenic spots. Under the background of the new era, bilingual language landscape has become more popular either in our real life space or in virtual space. However, problems in English translation of linguistic landscape are found here and there, which seriously affects the effect of tourism services and cultural transmission, and even causes a negative effect on the construction of the city's image. From the perspective of creative treason, this paper analyzes the current status of English translation of language landscape by using English translation of scenic spots names as examples, and explores the strategies of English translation of language landscape and expressions that are easily accepted by international tourists, so as to improve the quality of language services, strengthen Chinese culture transmission and achieve cross-cultural communication.
\end{abstract}

\section{Subject Areas \\ Culture, Linguistics}

\section{Keywords}

Scenic Spots, Language Landscape, Creative Treason

\section{1. 引言}

旅游景点语言景观是旅游公共服务的重要组成部分, 是国际旅游目的地 语言环境、人文环境的重要组成部分 [1]。目前, 我国旅游景点语言景观多以 汉英双语为景观主体语言。汉英双语语言景观中的英译发挥着重要的外宣推 介作用, 是西方游客了解当地旅游景点服务和历史文化的重要窗口, 往往起 着画龙点睛的作用。语言景观英译质量直接影响着当地景点的对外形象宣传 和西方游客的旅游体验。因此, 规范、合理、易懂的英译文很有必要。然而, 
目前我国旅游景点的翻译现状不容乐观, 译文质量良莠不齐、景点名称生硬 直译、误译随处可见。本文针对旅游景点名称翻译现状问题, 根据 “创造性 叛逆” 原则, 提出旅游景点名称翻译的有效策略。

\section{2. 旅游景点名称英译现状}

尽管我国已经成为世界最重要的旅游目的地之一，然而国内许多旅游景 点语言景观在语言表述上存在不少错误，不仅严重影响了我国的国际形象， 同时也影响了各国人民之间的交流与沟通。当前, 我国旅游景点语言景观翻 译主要存在以下两个方面的问题: 第一, 缺乏统一规范, 译名不统一; 第二, 生硬直译，文化意象丢失。

\section{1) 译名不统一}

以陕西省旅游景点为例, 同一旅游景点多种译名现象尤为严重, 如以下 四个表格所示:

如上表所示, “大雁塔” 的英译名有 9 个(见表 1), “黄帝陵” 有 7 个(见 表 2), “中书省” 有 2 个(见表 3), “殿中省” 有 2 个(见表 4)。其中, “中 书省” 和 “殿中省” 在同一景点的指示牌中，英译名竟各不相同。旅游景点 名称，作为旅游宣传的专有名词，应属于术语类型，英译时应尊重原语术语 及官方源信息进行规范、准确表达最为重要。一个旅游景点的英译名如同景 点名片, 好的景点译名不但可以帮助提升景点的国际知名度, 还可以让国际 游客通过名称了解景点的文化内涵[2]。然而, 陕西省作为我国文明的发祥地 和文化遗产旅游大省，其景点的英译名称缺乏规范统一，会给游客带来极大 的不便和困扰，严重影响游客体验，甚至有损国家形象。

\section{2) 生硬直译}

我国一些旅游景点是根据神话传说或历史传说而命名的。很多景点的名 称都蕴含着深厚的文化底蕴。如果在翻译景点名称时, 忽略其中的历史文化 因素, 直接按照字面意思生硬直译, 国外游客就无法理解其中的文化内涵,

表 1. 大雁塔景点英译名[2]

\begin{tabular}{|c|c|c|}
\hline 名称 & 英译名 & 出处 \\
\hline \multirow{9}{*}{$\begin{array}{l}\text { 大 } \\
\text { 雁 } \\
\text { 塔 }\end{array}$} & 1) Dayanta & 西安绕城高速路口 \\
\hline & 2) Dayan Tower & (同一出口两个不同牌子) \\
\hline & 3) Greater Wild Goose Pagoda & 旅游局宣传册 \\
\hline & 4) Giant Wild Goose Pagoda & 维基百科 \\
\hline & 5) Big Wild Goose Pagoda & 旅游教材、旅游社网站 \\
\hline & 6) Big Goose Pagoda (Da Yan Ta) & 国外出版物 [3] \\
\hline & 7) The Big Wild Goose Pagoda & 陕西省旅游局官方网站: \\
\hline & 8) The Greater Wild Goose Pagoda & 同一网站, 图片与内容名称不一致 \\
\hline & 9) Big Goose Pagoda & $\begin{array}{c}\text { 国外旅游网站: } \\
\text { www.lonelyplanet.com }\end{array}$ \\
\hline
\end{tabular}


表 2. 黄帝陵景点英译名[2]

\begin{tabular}{llc}
\hline 名称 & \multicolumn{1}{c}{ 英译名 } & 出处 \\
\hline & 1) The Mausoleum of the Yellow Emperor & \\
2) Yellow Emperor Mausoleum & 旅游局宣传册 \\
黄 & 3) Huangdi (Yellow Emperor) Mausoleum \\
\cline { 2 - 3 } 帝 & 4) Yellow Emperor's Mausoleum & 教材 \\
\cline { 2 - 2 } & 5) Mausoleum of the Yellow Emperor & 维基百科 \\
\hline 6) Mausoleum of Yellow Emperor & 省、市旅游局网站 \\
\hline 7) The Yellow Emperor's Tomb & 国家旅游局网站 \\
\hline
\end{tabular}

表 3. 中书省景点英译名

\begin{tabular}{ccc}
\hline 名称 & 英译名 & 出处 \\
\hline 中书省 & Zhong Shu Sheng & 大明宫国家遗址公园指示牌 \\
& Central Secretariat & \\
\hline
\end{tabular}

表 4. 殿中省景点英译名

\begin{tabular}{ccc}
\hline 名称 & 英译名 & 出处 \\
\hline 殿中省 & Dianzhong Ministry & 大明宫国家遗址公园指示牌 \\
& Dian Zhong Sheng & \\
\hline
\end{tabular}

导致文化意象丢失, 造成跨文化交际障碍。例如, 佛教建筑大雁塔, 其旅游 官网上将 “大雁塔” 英译为 “The Big Wild Goose Pagoda”, 这一英译名看似 忠实, 实则属于生硬直译。原来, 相传大雁塔的命名与玄奘西行求法有关, 而佛塔本身不论是建筑形式或功能均与 “雁” 无关; 此外, 在国外 “Goose” 往往寓意着 “笨鸭、笨我” 的意思, 而在我国雁指冬候鸟, 显然, 此 “雁” 非彼 “雁”。再如, 张家界景点的象形石峰一一千里相会, 相传东海龙宫小 龙女常来此地观赏风景, 偶然结识了峨眉山远道而来的后生，二人情投意合。 龙王知道此事之后, 恼差成怒, 多次将二人拆散, 但小龙女想方设法与情人 相会。龙王一气之下，将二人点化成石，便成石峰 “千里相会” [4]。原来追 根述源, 中国婚姻讲究门当户对、贫富差距、社会地位等; 而西方注重情投 意合、两情相悦。此处英译名为 Meeting from Thousand-li away 看似字字对 应, 忠实原文, 但国外游客看了不得要领。

\section{3. 创造性叛逆的研究视角}

“创造性叛逆”，是法国文学社会家埃斯皮卡于 1958 年在其著作《文学 社会学》一书中提出来的, 他认为 “翻译总是一种创造性的叛逆”, 因为它 赋予作品一个崭新的面貌, 使之能与更广泛的读者进行一次崭新的文学交流; 它不仅延长作品的生命，而且又赋予它第二次生命 [5]。但埃斯皮卡并没有对 
“创造性叛逆” 做出严格界定和详细阐释。1999 年, 谢天振在其著作《译介 学》中引入这一概念, 他指出, 创造性叛逆是翻译过程中, 译者在某种明确 的再创作动机驱使下完成的创造性翻译活动, 认为 “叛逆” 是 “译者为了达 到某一主观愿望而造成的一种译作对原作的客观背离”。主要表现为有意识 型和无意识型两种, 具体有四种形态: 个性化翻译、误译与漏译、节译和编 译、转译和改编[6]。此后, 国内学者进一步将 “创造性叛逆” 运用于一般的 翻译研究。如, 许钧通过对 “创造性叛逆” 这一命题的重新阐释, 试图在 “忠 诚” 和 “叛逆” 两极中找寻翻译的主体性, 协调两者的矛盾。他认为语言符 号与意义之间不是一种固定的关系, 意义的生成依赖于多方面因素, 这就打 破了仅追求语言形式简单对应的传统做法。他将 “忠诚” 和 “叛逆” 比喻为 翻译的双重性格, 并指出愚笨的 “忠诚” 可能会导向 “叛逆”, 而巧妙的 “叛 逆” 可能会显出 “忠诚” [7]。创造性叛逆兼具 “偏离” 和 “传承” 双重含义。 它不仅仅是对原作的简单偏离, 同时也在目标语语境中传承原作之意, 不断 丰富原作。其特殊性在于脱离原有语境而遵循目标语语境中的模式进行再创 作 $[8]$ 。

“创造性叛逆” 这一概念虽然源于文学翻译领域, 但 Gaballo 认为, 由于 任何文本的翻译均存在不同程度的创造性, 所以创造性翻译并不是某个领域 翻译(如诗歌、计算机游戏或广告业)的特权, 严肃如法律文本有时也需要创 造性翻译 [9]。语言景观的语言特点和文体特点决定了原文和译文存在一定的 差异, 因此 “创造性叛逆” 可以用来指导语言景观翻译。

\section{4. 创造性叛逆视角下的景点名英文译写}

《公共服务领域英文译写规范》第三部分(2017) [10]规定了旅游服务领域 英文翻译和书写的相关术语和定义、翻译方法和要求、书写要求等, 为我国 旅游景点英文改写提供了切实可行的指导原则。该规范规定旅游景点改写标 准：1）山、河、湖等地名应使用汉语拼音, 对外服务中需要英文予以解释的, “山”一般用 Mountain 或 Hill 解释, 已经习惯使用 Mount 的可沿用。如黄 山译作 Mount Huangshan；2) 寺、庙应区分不同的情况, 采用不同的译法: 佛教的寺, 以及城隍庙、太庙等译作 Temple; 清真寺译作 Mosque; 3) 道教 的宫、观译作 Daoist Temple；4）塔应区分不同的情况，采用不同的译法：佛 教译作 Pagoda；舍利塔译作 Stupa 或 Pagoda；5）亭、台、楼、阁、榭、阙等 与专名一起使用汉语拼音拼写。

景点语言景观是景点的名片, 富含历史文化元素。因其受英汉语言形式、 文化元素、认知特点和思维方式差异等诸多因素的影响, 可借助 “创造性叛 逆” 这一概念, 实现语言景观想要传达的信息。创造性叛逆表现形式包括有 意识型和无意识型, 鉴于后者是因为译者对原文对语言内涵或文化背景知识 缺乏了解或大意疏忽而造成的, 这种叛逆的结果往往会产生负效应, 故本文 不讨论无意识型叛逆。景点语言景观翻译的有意识型创造性叛逆主要表现在 以下几个方面:

\section{1) 创造性译写结合}

在旅游景点翻译中, 翻译的首要目的就是引发游客兴趣, 让游客了解当 
地的人文景象、历史文化等进而实现跨文化交际的目的。我国旅游景点大多 都源自神话传说, 具有浓厚的历史文化底蕴, 因此景点语言景观翻译大多采 用创造性译写策略, 即译语文化 “屈从” 原著文化的现象 [11], 在创造性译写 策略, 音译的方法值得提倡, 采用原语的拼音作为译文, 以保留原有文化的 风味特点, 使原文化精髓得以发扬光大。

例 1: 大雁塔

原译文: The Big Wild Goose Pagoda

建议译文: Dàyàn Pagoda

大雁塔, 位于陕西省西安市南郊的大慈恩寺内, 是我国佛教建筑艺术杰 作, 全国重点文物保护单位, 国家 $5 \mathrm{~A}$ 级旅游景点。大雁塔的命名与玄牀西行 求法有关, 佛塔本身不论是建筑形式或功能都和 “雁” 无关。玄裝在《大唐 西域记》中记载了他在印度所闻僧人埋雁造塔的传说。据《大唐西域记》记 载, 相传摩揭陀国(今印度比哈尔邦南部)一个寺院内的和尚信奉小乘佛教, 吃三净食(即雁、鹿、犊肉)。一天, 空中飞来一群大雁, 一位僧人见到群雁, 向天说道: “菩萨啊, 今天和尚们都没有东西吃了, 您那么神通广大, 应该 知道我们的苦呀! ”话音刚落, 突然就有一只大雁坠死在这位僧人面前, 他 惊喜交加, 遍告寺内众僧, 大家都认为这是如来佛在教化他们。于是就在雁 落之处, 以隆重的仪式葬雁建塔, 并取名 “雁塔” 。大雁塔是玄牀仿照印度 的雁塔而建, 沿袭印度塔之原名, 塔名前加一 “大” 字是代表大乘佛教之意, 大雁塔是中华文化发展的历史长河中与外来文化融合的历史见证, 也由此可 见, 塔名是跟 “雁” 有关。

在各种翻译版本中, 按字面一一对应的 “The Big Wild Goose Pagoda” 版 本使用较为广泛。但这一译本却引起国内外的热议。根据国家发改委发布的 《公共服务领域英文译写规范》, 佛教的塔译作 Pagoda, 故争议的焦点在于 “大雁”一词的翻译上。“Wild Goose”一词在新版的牛津字典中并不能查到 其确切的含义。原来, 英文的 “Goose” 只是欧洲人对野生鸭类与鹅类的一种 俗称, 往往寓意着 “笨鸭、笨鹅” 的意思, 在我国, 雁指冬候鸟, 常见的有 鸿雁、灰雁、豆雁、白额雁等。英译为 “The Big Wild Goose Pagoda”, 这与 大雁塔的藏经功能相悖, 容易造成误解。所以, 大雁塔的翻译应采取译写结 合的方法, 音译为 “Dàyàn Pagoda”。鉴于汉语拼音都有其固有的声调, “大 雁” 音译为 Dàyàn, 既有利于向国外游客传播汉语拼音, 又保留了 “大雁” 在我国原有的文化的风味，符合名从主人的翻译原则。

例 2: 黄帝陵

原译文: The Mausoleum of Yellow Emperor

建议译文: The Mausoleum of Huángdì Emperor

黄帝陵是中华民族始祖轩辕黄帝的陵寝。在皇帝陵的翻译中, 主要在于 如何翻译 “皇帝” 和 “陵”。首先, “陵” 的翻译相对简单, “mausoleum” 为帝王陵墓, “tomb” 为普通坟墓, 此处的 “陵” 应翻译成 “mausoleum”。 其次, “皇帝” 应如何翻译? 查阅国外大量文献发现, “皇帝” 皆译为 “Yellow 
Emperor”。皇帝是我国帝制时期最高统治者的称号。中国最早所谓的“皇帝”, 是对 “三皇五帝” 的统称, 三皇指天皇、地皇和人皇, 是传说中的三个古代 帝王; “帝” 原指宇宙万物至高无上的主宰者, 即天帝, 后来许多国家混战, 各自称帝, 出现西帝、东帝、中帝、北帝等, 使天上的 “帝” 来到人间, 成 为超越 “王” 的人间尊号 (也有说是部落时期的皇帝、炎帝、虫尤等)。秦始 皇统一中国后, 自认为 “德兼三皇, 功盖五帝”, 将 “皇” “帝” 这两个人 间最高的称呼结合起来作为自己的称号, 从此天子称为皇帝。另外, “黄色” 在我国古代是极其高贵的颜色, 为皇家御用; 而十九世纪末英国的低俗小说 往往用黄色封面, 在英语语境中, “Yellow” 具有色情之义。显然 “Yellow Emperor” 并不能传递 “皇帝”之意。鉴于 “皇帝”一词在我国历史上的文化 意象和其名称的约定俗成性, 应对原作进行偏离, 采取译写结合的方法, 音 译为 “The Mausoleum of Huángdi Emperor” 。通过创造性译写结合, 既在英 语语境中保留了 “皇帝” 一词的文化意象和风味特点, 使我国文化精髓得以 发扬光大, 又向国外游客传播了汉语拼音及其声调, 以实现跨文化交际的目 的。

\section{2）创造性增译}

创造性增译, 就是在翻译过程中, 有意识增加原文中无其形而有其意的 信息, 增译原文所无之辞格而丰姿凸现[12], 使译文与原文达到形式、功能上 的对等, 从而使游客充分了解景点的文化内涵。

例 3: 千里相会

原译文: Meeting from Thousand-li away

建议译文: Dating after Long Isolation

“千里相会”, 讲述了一对相爱恋人被粗暴干涉而被迫分开, 历经千难 万苦后终于相聚的故事。用 “千里相会” 来命名一座山峰, 突出了一对相爱 恋人突破层层阻挠、历经千辛万苦奔波千里在此相聚的炽热情感。原文 “Meeting from Thousand-li away” 采用直译策略, 游客无法体会其中男女恋 人冲破层层阻挠、其间经历千辛万苦的文化意蕴。运用创造性手段, 增加了 原文所蕴含的文化内涵, 将其实化, 译作 “Dating After Long Isolation”, 突 出了恋人被迫分离后又重聚的情感, 而该景点的历史传说故事也通过译名立 马呈现在国外游客面前, 实现了跨文化交际的目的。

例 4: 梨园

原译文: Li Yuan

参考译文: Lí Yuán (Royal Opera School)

梨园位于陕西省西安市大明宫太液池一带, 是我国历史上第一所皇家音 乐艺术学校, 被尊奉为中国戏曲艺术的鼻祖。唐中宗执政时期, 梨园只是皇 家禁苑中的一个果木园, 供皇室宴饮游乐的场所。后来经唐玄宗李隆基的大 力倡导, 梨园的性质起了变化, 由一个单纯的果木园圃, 逐渐成为唐代的一 座 “梨园子弟” 演习歌舞戏曲的梨园, 成为我国历史上第一座集音乐、舞蹈、 戏曲的综合性 “艺术学院” [13]。梨园原译名为 “Li Yuan”, 仅通过音译的 
方式无法体现梨园的历史由来和功能, 通过创造性增译, 借助添加注释, 向 游客呈现了梨园的历史由来, 将跨文化意识充分体现在景点翻译中, 易于游 客理解。

\section{3) 创造性改写}

创造性改写就是指在翻译过程中, 根据中西方语言表达差异或文化意象 的不同对原语进行适当 “偏离” , 运用创造性手段进行一定程度的改写以适 应目标语言的语言表达习惯, 便于读者理解。

例 5: 采薇路

\section{英译名：Romance Road}

采薇路位于我国首个诗经主题特色小镇一陕西省西安市的旅游景点诗经 里, 是一条在园林里用砖块铺就的双排并行小路。《采薇》选自《诗经》, 是一首在民间广为传唱的戍卒返乡诗歌, 唱出了从军将士艰辛的行军生活和 思乡归家的情怀。另有鲁迅小说《采薇》以商代小国孤竹国的公子伯夷和叔 齐为原型。周武王建立周朝后, 他们拒绝吃周朝的粮食, 在首阳山上采摘薇 菜充饥, 终饿死在首阳山。不论是诗经里还是鲁迅笔下, 采薇的故事里都蕴 含了人们的思乡爱国的伟大情怀, 传递着中国优秀传统文化。今天旅游景点 诗经里的采薇路借用《诗经》和鲁迅笔下的 “采薇” 一词, 意在让受众漫步 “采薇路”上时能深刻体会采薇故事, 继承中国优秀传统文化。不可否认, Romance Road 这一英译名造成了原语文化缺位, 无法传递诗经及鲁迅笔下的 采薇故事。但从另一方面看, 译者结合时代背景及实地语境, 大胆创新, 进 行适当 “偏离”, 将采薇路译为 Romance Road, 意在传达浪漫爱情之意。通 过创造性改写, 浪漫爱情的文化意象即可浮现在目标语受众的面前。

\section{4) 创造性删减}

旅游景点景点名称短小精悍, 为了使其译名表述更加准确、有效, 在翻 译时, 就有必要对一些旅游景区的修辞成分进行创造性删减, 以有效传递景 点文化内涵。

例 6: 大唐西市

原译文: Da Tang West Market

建议译文: Tang West Market

“大唐西市” 是西安市政府形成的一个以文化为主线, 以丝路风情和旅 游会展为特色的文化产业项目, 是陕西非公有制经济发展文化产业的成功范 例, 也是西安的城市新名片和陕西旅游的新亮点。鉴于中西语言表达习惯差 异, 此处的 “大” 属于修辞成分, 无实际含义, 英译时应采用创造性删减原 则, 去掉修饰成分 “大”, 译为 “Tang West Market”, 以符合国外游客的语 言表达习惯和审美习惯。

\section{5. 结语}

旅游景点大多源于历史故事、神话传说, 在翻译时, 如果没有统一规范 或者采取生硬直译翻译方法, 会导致文化意象错位、丢失, 难以达到跨文化 
交际的目的, 影响国外游客对旅游景点风景的欣赏和文化意蕴的理解。本文 从创造性叛逆的视角, 以旅游景点名称为例, 案例分析我国旅游景点语言景 观的英译现状，运用创造性译写结合、创造性增译、创造性改写、创造性删 减等创造性翻译策略, 但本文仅是作者对语言景观英译策略的探索, 期待更 多学者投入旅游景点英译研究, 提升语言服务质量, 加强中国文化对外传播, 实现跨文化交际的目的。

\section{基金项目}

1) 大西安旅游文化与城市语言景观表达深度融合研究(2020K02), 主持, 省社科基金，在研。

2) 大明宫遗址虚拟语言景观文化表达研究，陕西省社科联，主持，2021 年 6 月, 在研。

\section{Conflicts of Interest}

The authors declare no conflicts of interest.

\section{References}

[1] 戴宗显, 吕和发. 公示语汉英翻译研究一一以 2012 年奥运会主办城市伦敦为例 [J]. 中国翻译, 2005, 26(6): 38-42.

[2] 乌永志. 文化遗产类旅游景点名称汉英翻译规范研究[J]. 外语教学, 2012，33(2): 93-97.

[3] Bonavia, J. (2004) The Silk Road: Xi'an to Kashga. Odyssey, Hong Kong.

[4] 汤敬安, 余叶子. 旅游景点英译公示语的认知研究——以张家界为例 [J]. 中国科 技翻译, 2019, 32(3): 50-52.

[5] 埃斯卡皮. 文学社会学[M]. 合肥: 安徽文艺出版社, 1987.

[6] 谢天振. 译介学 [M]. 南京: 译林出版社, 2013.

[7] 许钧. “创造性叛逆”和翻译主体性的确立[J]. 中国翻译, 2003(1): 8-13.

[8] 范若恩, 刘利华. 偏离叛逆/传播传承一一“创造性叛逆”的历史语义和翻译文学 的归属[J]. 人文杂志, 2021(4): 1-11

[9] Gaballo, V. (2012) Exploring the Boundaries of Transcreation in Specialized Translation. ESP Across Cultures, 2012, 111.

[10] 中华人民共和国国家质量监督检查检疫总局/中国国家标准化管理委员会. GB/T30240.3 公共服务领域英文译写规范第 3 部分: 旅游[S]. 北京: 中国标准出 版社, 2017.

[11] 谢天振. 论文学翻译的创造性叛逆[J]. 外国语(上海外国语学报), 1992(1): $32-39+82$.

[12] 杨全红. 也谈汉英公示语的翻译[J]. 中国翻译, 2005(6): 43-46.

[13] 南翔宇. 华清池: 重重历史堆砌的殿堂[J]. 前进论坛, 2005(3): 38-40. 


\section{Appendix (Abstract and Keywords in Chinese)}

\section{创造性叛逆视角下旅游景点名称英译研究}

摘要: 国家语言文字工作委员会要求旅游景点须呈现中英双语语言景观。新 时代背景下, 我国绝大部分旅游景点中英双语语言景观已呈普遍现象, 并以 多模态形式对外宣传。然而旅游景点景观语的英译问题也普遍存在, 严重影 响旅游服务和文化对外传播效果, 甚至造成国家形象负效应。本文拟从创造 性叛逆的视角, 以旅游景点名称为例, 案例分析我国旅游景点语言景观的英 译现状, 探索语言景观英译策略及易于国际受众接受的表达形式, 提升语言 服务质量, 加强中国文化对外传播, 实现跨语言、跨文化交际的目的。

关键词: 旅游景点, 语言景观, 创造性叛逆 